\title{
Synchronization of Sound Sources
}

\author{
Markus Abel, Karsten Ahnert, and Steffen Bergweiler ${ }^{*}$ \\ Department of Physics and Astronomy, Potsdam University, \\ Karl-Liebknecht-Str. 24, D-14476, Potsdam-Golm, Germany
}

(Dated: November 2, 2018)

\begin{abstract}
Sound generation and -interaction is highly complex, nonlinear and self-organized. Already 150 years ago Lord Rayleigh raised the following problem: Two nearby organ pipes of different fundamental frequencies sound together almost inaudibly with identical pitch. This effect is now understood qualitatively by modern synchronization theory (M. Abel et al., J. Acoust. Soc. Am., 119(4), 2006). For a detailed, quantitative investigation, we substituted one pipe by an electric speaker. We observe that even minute driving signals force the pipe to synchronization, thus yielding three decades of synchronization - the largest range ever measured to our knowledge. Furthermore, a mutual silencing of the pipe is found, which can be explained by self-organized oscillations, of use for novel methods of noise abatement. Finally, we develop a specific nonlinear reconstruction method which yields a perfect quantitative match of experiment and theory.

PACS numbers: 43.25.+y, 05.45.Xt, 07.05.Kf, 07.05.Tp
\end{abstract}

Introduction. In a seminal publication Lord Rayleigh reported on experiments involving two organ pipes of close pitch, positioned next to each other. He observed the following peculiar behavior: alone, each pipe sounded with its own natural frequency. Together they sounded in perfect unison and almost reduced one another to silence [1]. This phenomenon can be described by synchronization theory, a general nonlinear principle with striking applications in the natural sciences including physics, chemistry, neurology or biology [2].

Here, we focus on an organ pipe as i) prototypical for aero-acoustical sound generation, ii) paradigmatic for synchronization of coupled oscillators and iii) a beautiful musical instrument for which we present a mathematical model. By driving one organ pipe sinusoidally by an electric speaker we can demonstrate in great detail the importance of nonlinear effects in sound generation and -interaction 3 in contrast to linear response theory 4 conventionally applied for musical instruments.

The general description of the dynamics of an organ pipe is given by the compressible Navier-Stokes Equations with suitable boundary conditions. One can solve the equations numerically [5], or investigate them analytically [6]. Both ways reproduce different aspects of sound production. Here, we are interested in the interaction of an organ pipe with a sound source. Then, it is of advantage to model only the relevant characteristics in terms of reduced models [7. Such an elementary model allowing for complex dynamics is given by an autonomous oscillator [2, 8, which includes an oscillatory unit, energy supply, and energy loss by radiation and damping.

Let us identify these units in the organ pipe. Energy is supplied steadily by the wind system through the pipe foot and establishes a turbulent vortex street. Each time

\footnotetext{
*corresponding author: markus.abel@physik.uni-potsdam.de
}

a vortex detaches, a pressure fluctuation enters the resonator, inside which characteristic waves are selected, and radiated at the pipe mouth by an oscillating airsheet 7, 9. In our model, this air-sheet constitutes the basic oscillating unit. Inside the resonator Sound Pressure Levels (SPLs) up to $160 \mathrm{~dB}$ can occur, such that viscous damping contributes in energy dissipation. External acoustical fields couple to the system through the air-sheet, possibly described by a (nonlinear) acoustical admittance [10. By Lighthills analogy [6], a coupling by the turbulent vortex street is expected to be of lower order.

The above scenario can be described by a reduced, twodimensional model for the oscillatory unit $\xi$ :

$$
\ddot{\xi}-g(\xi, \dot{\xi})=0,
$$

where the function $g(\xi, \dot{\xi})$ contains the above mentioned ingredients and with the condition that a limit-cycle solution of frequency $\nu_{0}$ exists. Then, with $\xi=A(t) \cdot e^{i \phi(t)}$ the phase $\phi$ and the amplitude $A$ are well defined. An external driving enters on the right hand side of Eq. (1). Corresponding to the experiment, sinusoidal driving is used: $\varepsilon \sin \left(2 \pi \nu t+\phi_{0}\right)$ with $\varepsilon$ the coupling strength. Here, one assumes that an oscillator represents the basic physics of the pipe with regard to sound generation and synchronization - typically, the oscillating air sheet 7. Because the air sheet is the source of sound radiation, the measurement at the microphone can be taken as the state of the oscillator (with a phase shift accounting for distance).

Close to the limit cycle the amplitude is slaved by the phase, allowing the description in terms of the phase difference, $\Psi$, between driving and oscillator

$$
\dot{\Psi}=-2 \pi\left(\nu-\nu_{0}\right)+\varepsilon q(\Psi) .
$$

The parameters are driving frequency $\nu$, and coupling $\varepsilon$. The study of the parameter plane $(\nu, \varepsilon)$ yields triangularshaped synchronization regions, the well-known ArnoldTongues [11. 


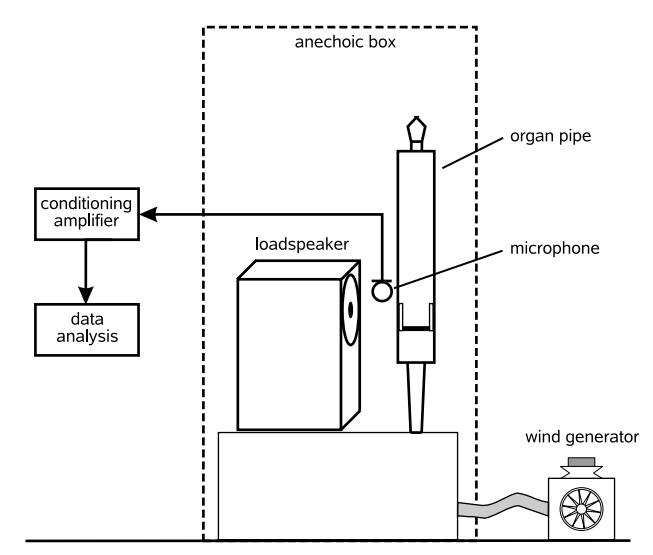

FIG. 1: Sketch of the experiment. Pipe and loudspeaker stand side by side, the signal is measured by a microphone with equal distance to both sound sources.

In order to determine Eq. (1) directly from data, we have elaborated a numerical method based on embedding theory [12] which allows for a reconstruction and comparison of the characteristics of model and data. We recover the power spectra and synchronization properties of the organ as an acoustical systems. This opens the way for a detailed theoretical investigation of the system and hints to how a model can be derived from first principles.

The experiment. The setup of the experiment is sketched in Fig. 1. The pipe 13. was wooden and closed at the upper end, tuned at $\nu_{0}=686 \mathrm{~Hz}$. It was driven by an especially fabricated miniature organ [13] with a blower connected to the wind-belt and further by flexible tubes to the wind-chest. Measurements took place inside a suitable anechoic box. The loudspeaker for the external, driving sound signal, was positioned side-byside to the organ-pipe, cf. Fig. 1. The emitted signals were registered at a distance of $16 \mathrm{~mm}$ to either pipe and speaker, allowing for constructive or destructive interference of the superposed signals. To ensure that the phase of the pipe is correctly detected, we carried out simultaneous control measurements inside and outside the pipe and at the microphone - all results were consistent.

To explore the coupling-detuning plane $(\nu, \varepsilon)$, the loudspeaker $S P L$ and frequency were varied separately; the first between $+10 \mathrm{~dB}$ and $-50 \mathrm{~dB}$ relative to the reference signal of the organ pipe in steps of $2 \mathrm{~dB}$, the latter according to the size of the synchronization range. To determine the minimal achievable resolution in $\nu$, we consider the sources of variations of the pipe's frequency. The wind pressure was $700 \pm 9 \mathrm{~Pa}$, giving a frequency variation of $\pm 0.1 \mathrm{~Hz}$ at the scale of seconds; the temperature varied with the circadian rhythm at $292 \pm 1 \mathrm{~K}$ with a resulting variance of $\simeq 2 \mathrm{~Hz}$ [14]. This slow change did not affect an individual run with fixed $S P L$, however, runs with different $S P L$ were adjusted to the circadian variation. We measured the synchronization range down to the maxi-



FIG. 2: Synchronization plot: if the detuning of pipe and loudspeaker is small enough, frequency synchronization is found as a plateau. Within the synchronization region, the phase difference $\Psi$ varies over an interval of size $\pi$, where the measured $S P L$ shows a sharp minimum for $\Psi=\pi$. Top $\left(S P L_{\text {Speaker }}=S P L_{\text {Pipe }}\right):$ the frequency shows a very nice plateau with a saddle-node bifurcation; for $\Psi=\pi$ negative interference is observed by a decrease of $6 \mathrm{~dB}$. Bottom $\left(S P L_{\text {Speaker }} \simeq S P L_{\text {Pipe }}+10 \mathrm{~dB}\right)$ : the transition from synchronization is quite sharp, indicating strong nonlinearities. The amplitude decrease can be explained by synchronization with an additional Helmholtz-resonator-like behavior of the pipe, deepening the $S P L$ gap to $15 \mathrm{~dB}$. The maximum is obtained by addition of the amplitudes to $12 \mathrm{~dB}$.

mal resolution of $0.1 \mathrm{~Hz}$ as set by the wind supply; below noise destroys synchronization. With respect to noise, we enhanced the signal-to-noise ratio was enhanced by longtime averaging, such that irregular phase slips are leveled out and a synchronization region can be obtained even for very small driving. This was achievable by heavily automatized measurements of a total duration of about 3 weeks.

We investigated two acoustically relevant characteristics: the frequency difference, $\Delta \nu / \nu_{0}=\left(\nu_{\text {Speaker }}-\right.$ $\left.\nu_{\text {Pipe }}\right) / \nu_{0}$, and the spectrum of the measured loudspeaker signal. Their dependence on the detuning is shown in Fig. 2 for two exemplary relative driving strengths, $0 \mathrm{~dB}$ and $10 \mathrm{~dB}$. Note, that $\nu_{\text {Pipe }}$ is the frequency of the driven pipe which might be different from its natural frequency.

The transition to synchronization can be seen from the graph for the frequency difference. For equal $S P L$ and below (upper panel in Fig. 2), a saddle node bifurcation is found, as predicted theoretically [2, 15]. For couplings stronger than $0 \mathrm{~dB}$, the bifurcation tends to become very sharp, indicating that the weakly nonlinear approximation 2] breaks down (lower panel Fig. 2).

In the graphs for the amplitude, one recognizes two effects: synchronization and resonance. Due to synchronization the phase shift between the two emitted signals 


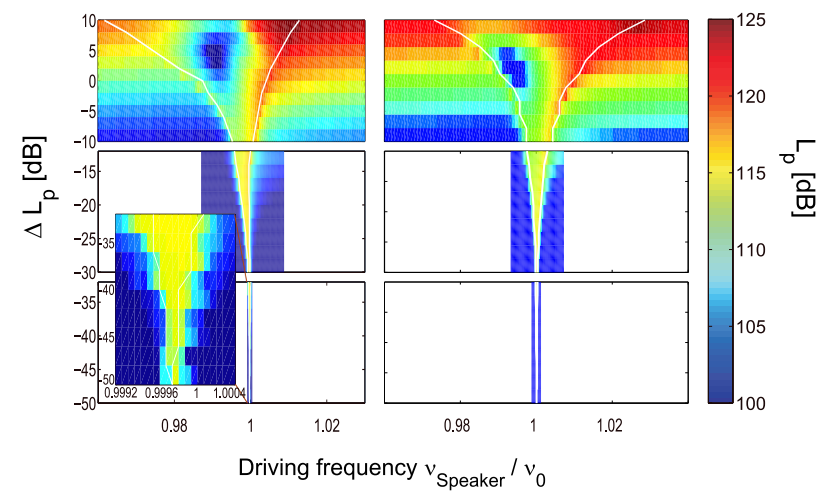

FIG. 3: Arnold tongue measured over approximately 3 decades. The left plot shows the Tongue obtained from the experimental data, while the right one shows the reconstructed tongue. The color coding corresponds to the amplitude measured at the microphone position at the loudspeaker frequency $S\left(\nu_{\text {Speaker }}\right)$. To guide the eye, the synchronization region is marked by a straight line. The synchronization edge grows linearly with the coupling, seen as a logarithmic bend in the used semi-log plot. The lowest possible frequency resolution, $0.1 \mathrm{~Hz}$, corresponds to the variation of the frequency produced by the wind supply, shown by the inset. The reconstructed Arnold tongue is much more symmetric. This indicates again strong nonlinearities. The coincidence between experiment and model is almost perfect in the low-coupling region.

varies within an interval of size $\pi$; as for Helmholtzresonance, the loudspeaker signal is phase-shifted by $\sim \pi$ and re-emitted by the pipe. From synchronization alone, the superposition of the signals of speaker and pipe can only vary within $S P L_{\text {Speaker }} \pm S P L_{P i p e}$; with resonance much weaker amplitudes result, as seen in Fig. 2. This result implies a novel way of sound reduction, where the reductor acts as an active element, adjusting the frequency without any external control.

The full variation in parameter space is shown in the left panel of Fig. 3 by an Arnold Tongue, here plotted logarithmically due to the enormous coupling range investigated. The color coding corresponds to the the spectral power of the loudspeaker frequency $S\left(\nu_{\text {Speaker }}\right)$, measured at the microphone. We varied the amplitude of the loudspeaker from $+10 \mathrm{~dB}$ to $-50 \mathrm{~dB}$, relative to the $S P L$ of the organ pipe, in steps of $2 \mathrm{~dB}$. The range covers 3 decades - the widest range ever measured in synchronization experiments. The synchronization edges are marked in Fig. 3 by white lines. Clearly, the linear shape is bent due to the semi-log plotting.

Reconstruction of the dynamical system. We do not have access to the "state" of the oscillating air-sheet, but we know the recorded $S P L, x(t)$, of the organ pipe. By embedding theory we can infer a differential embedding $(x, \dot{x}, \ddot{x}, \ldots)$ with a maximal embedding dimension of three [12, 16. In this space there exists an equivalent to Eq. (1): $\ddot{x}-f(x, \dot{x})=0$. We reconstruct this equation step by step to further investigate its dynamical and predictive properties.

The data series consists of 110250 data points, with a sampling rate of $11025 \mathrm{~Hz}$. Normalization in space and time yields variance 0.5 and frequency 1 . The crucial computation of derivatives was accomplished by spectral smoothing [17]: i) Fourier transformation, ii) 8th order Butterworth-filtering with cutoff at $4.5 \nu_{0}$ to suppress noise amplification, and iii) back-transformation. If the cutoff is increased more harmonics enter the filtered time series, if the cutoff is too low too much information is filtered out, i.e. the necessary nonlinearities are suppressed. Thorough testing yielded that in our case a three dimensional embedding do not improve the results.

The unknown function $f$ is estimated by nonparametric regression, formulated as a minimization problem: $\|\ddot{x}-f(x, \dot{x})\|_{2} \stackrel{!}{=}$ min, with $\|\cdot\|_{2}$ the $l^{2}$-norm of the data vector. The unknown function $f$ is found by variation in function space, where we used, for the sake of computational simplicity, polynomials of order three [18, 19]; higher orders do not improve the model. Specifically, $f(x, \dot{x})=a_{0}+a_{1} x+a_{2} x^{2}+a_{3} x^{3}+a_{4} \dot{x}+a_{5} \dot{x}^{2}+a_{6} \dot{x}^{3}+$ $a_{7} x \dot{x}+a_{8} x^{2} \dot{x}+a_{9} x \dot{x}^{2}$ with $a_{0}=0.25, a_{1}=-0.92, a_{2}=$ $-0.18, a_{3}=-0.12, a_{4}=0.20, a_{5}=-0.33, a_{6}=$ $0.056, a_{7}=-0.015, a_{8}=-0.923, a_{9}=-0.072$. Note the striking similarity to the van der Pol oscillator with $f_{v d P}=-x+\dot{x}\left(1-x^{2}\right)$, reflected in the dominant coefficients $a_{1}$ and $a_{8}$. The latter is responsible for nonlinear damping, whereas energy is supplied by the constant and the $\dot{x}$-term. Other terms assist nonlinear damping and are indispensable to find the correct frequencies in the reconstructed system. Since the observables are not directly related to the physical driving (wind) and damping mechanisms we hesitate giving a complete physical interpretation.

Numerical stability analysis yields a repelling fixed point at $(0.254,0)$ and an attracting limit cycle, plotted in the inset of Fig. 4, which shows convincing coincidence with the filtered experimental data. For acoustical comparison, we compare the power spectra of pipe and reconstruction in Fig. 4. The positions of the harmonics are in perfect agreement, and their ratio coincides well.

Finally, the model is synchronized with an external, sinusoidal driving. For the coupled equation, we solve $\ddot{x}=f(x, \dot{x})+\epsilon \sin (\omega t)$, with the driving frequency $\omega$ and the coupling parameter $\epsilon=0.025 \cdot S P L_{\text {Speaker }}$. The latter relation describes the influence of the loudspeaker on the pipe with a factor 0.025 , determined in order to obtain optimal coincidence of experimental and reconstructed Arnold Tongue, see Fig. 3. Recently, a way to extract the coupling from data has been proposed [20]; in principle it can be obtained by a detailed analysis of acoustics and fluid dynamics at the pipe mouth.

Conclusion. Since the time of Lord Rayleigh the nonlinear interaction of acoustical sources is under discussion 1]. We highlight the acoustical effects of phase synchro- 

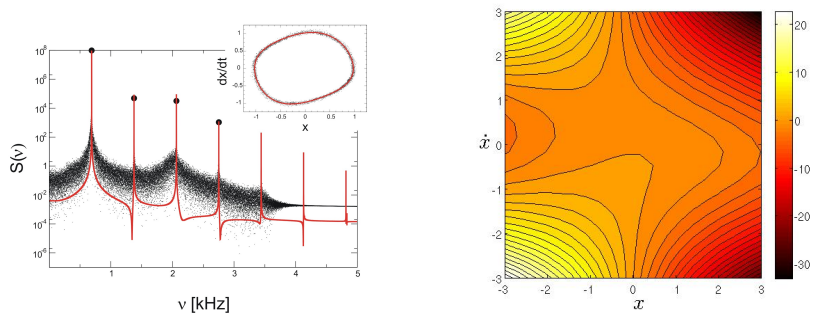

FIG. 4: Left: The power spectra of the measured (black) and the reconstructed signal (blue). The musical sound is reproduced quite nicely, as recognized by the coincidence of the spectra; for better visibility the maxima of the measured signal are shown by black dots. The inset shows the time series and its reconstruction in the embedding space, which coincide very well. Right: Contour plot of the function $f$ found by nonparametric regression, the color coding is shown by the colorbar on the right.

nization, experimentally realized by an organ pipe driven externally by a loudspeaker. The application of synchronization theory suggests a novel type of sound control, where the passive element adjusts its frequency exactly to the source. Since the system involved can be abstracted, we conclude that such a control can be applied to a diversity of situations, from musical instruments to noise reduction in vibrating systems, be it mechanical or hydromechanical.

With respect to synchronization, we found the deepest Arnold tongue ever seen experimentally, suggesting wind instruments as paradigmatic for synchronization. The results confirm the theory for small coupling; for large coupling we give experimental access to nonlinear correction terms to be analyzed further. To analyze the acoustical properties of the pipe we propose an autonomous oscillator, reconstructed from a novel type of data analysis. The agreement between model and experiment in terms of Arnold Tongues and power spectra is excellent - musical and synchronization characteristics are well reproduced. Conventional methods, as transfer functions, or admittance [21] do not allow such a direct interpretation

In this work acoustics is paired with nonlinear dynamics and data mining. Organ builders have developed complicated empirical rules to arrange organ pipes within a register to avoid synchronization effects. Our results do not only allow for an easy simulation of instruments, but as well development cycles and tuning of instruments could be enhanced. Noise reduction is possible by selforganization of two sound sources such that they interfere negatively - no external control is needed. The applications of this principle might be interesting for a variety of technically important situations.

Acknowledgments. We acknowledge inspiring discussion with A. Pikovsky and M. Rosenblum, and thank F. Spahn for many suggestions. The organ builder company
Schuke GmbH constructed the pipes and the wind supply system.

[1] J. W. S. Rayleigh. On the pitch of organ-pipes. Phil. Mag., XIII:340-347, 1882.

[2] A. Pikovsky, M. Rosenblum, and J. Kurths. Synchronization-A Universal Concept in Nonlinear Science. Springer, Berlin, 2001.

[3] N. H. Fletcher. The nonlinear physics of musical instruments. Rep. Prog. Phys., 62:723-764, 1999; N. H. Fletcher. Mode locking in non-linearly excited inharmonic musical oscillators. J. Acoust. Soc. Am., 64:156669, 1978.

[4] T. Rossing and N. Fletcher. The physics of musical instruments. Springer, New York, 1998.

[5] S. Succi. The lattice Boltzmann equation for fluid $d y$ namics and beyond. Oxford University Press, New York, US; J. C. Hardin and M. Y. Hussaini. Computational Aeroacoustics. Springer, Berlin, 1993.

[6] M. S. Howe. Theory of vortex sound. Cambridge texts in applied mathematics. Cambridge University Press, Cambridge, UK, 2003.

[7] B. Fabre and A. Hirschberg. Physical modeling of flue instruments: A review of lumped models. Acustica - Acta Acustica, 86:599-610, 2000.

[8] A. Nayfeh and D. Mook. Nonlinear Oscillations. John Wiley, New York, 1979.

[9] B. Fabre, A. Hirschberg, and A. P. J. Wijnands. Vortex shedding in steady oscillation of a flue organ pipe. Acustica - Acta Acustica, 82:863-877, 1996.

[10] S. Thwaites and N.H. Fletcher. Acoustic admittance of organ pipe jets. J. Acoust. Soc. Am., 74:400-408, 1983.

[11] V. I. Arnold. Cardiac arrhythmias and circle mappings[sup a)]. Chaos: An Interdisciplinary Journal of Nonlinear Science, 1(1):20-24, 1991.

[12] T. Sauer, J. Yorke, and M. Casdagli. Embeddology. J. Stat. Phys., 65(3/4):579-615, 1991.

[13] Alexander Schuke GmbH, 2004. URL http://www. schuke.com

[14] D. A. Bohn. Environmental effects on the speed of sound. J. Audio Eng. Soc., 36(4):223-231, 1988.

[15] M. Abel, S. Bergweiler, and R. Gerhard-Multhaupt. Synchronization of organ pipes by means of air flow coupling: experimental observations and modeling. J. Acoust. Soc. Am., 119(4):2467 - 2475, 2006.

[16] N. B. Tufillaro, P. Wyckoff, R. Brown, T. Schreiber, and T. Molteno. Topological time series analysis of a string experiment and its synchronized model. Phys. Rev. E, 51(1):164-174, 1995.

[17] K. Ahnert and M. Abel. Numerical differentiation: global versus local methods. Comput. Phys. Commun., 177(10): 764-774, 2007.

[18] M. Abel, K. Ahnert, S. Mandelj, and J. Kurths. Additive nonparametric reconstruction of dynamical systems from time series. Phys. Rev. E, 71:15203, 2005.

[19] G. Gouesbet and C. Letellier. Global vector-field reconstruction by using a multivariate polynomial $L_{2}$ approximation on nets. Physical Review E, 49(6):4955, 1994.

[20] B. Kralemann, L. Cimponeriu, M.G. Rosenblum, A.S. Pikovsky, , and R. Mrowka. Phase dynamics of coupled 
oscillators reconstructed from data. Phys. Rev. E, 77: $66205,2008$.

[21] T. Cox and P. D'Antonio. Acoustic Diffusers and Ab- sorbers: Theory, Design and Application. Taylor \& Francis, London, 2004. 